\title{
Intensification des échanges de chaleur dans les dissipateurs de microordinateurs
}

\author{
Abdelkader Aris ${ }^{1, *}$, Ahmed Guessab ${ }^{1}$, Medjahed Driss Meddah ${ }^{2}$ \\ ${ }^{1}$ Ecole Nationale Polytechnique d'Oran, Département de Génie Mécanique, Laboratoire LaRTFM, \\ BP 1523 El M'Naouar, 31000 Oran, Algérie \\ ${ }^{2}$ Centre Universitaire de Nâama, Département de technologie, BP 66 Nâama 45000, Algérie
}

\begin{abstract}
Résumé. Nous proposons dans cet article, l'étude de l'effet de variation de la géométrie de l'ailette sur le refroidissement d'un microprocesseur. Plusieurs paramètres géométriques ont été variés: nombre d'ailettes, largeur et hauteur du canal, épaisseur de la base du dissipateur et le débit d'écoulements. La modélisation 3D est effectuée sous le logiciel floworks et la technique de mesure par caméra infrarouge est utilisée.
\end{abstract}

\section{Introduction}

Les appareils électroniques et les ordinateurs ont connu une grande réduction de la taille accompagnée d'une croissance rapide de leurs fonctions. Si le refroidissement de ces systèmes devient une tâche difficile en raison de leur complexité, entraînant une augmentation de la chaleur produite, alors la surchauffe peut provoquer des dysfonctionnements. Afin d'éviter ce problème, il est nécessaire de maintenir leur température à moins d'une valeur critique $[1,2]$. Peu de travaux réalisés sur l'optimisation de l'amélioration des paramètres géométriques de micro-canaux : Steinke et Kandlikar [3] et Lee et Garimella [4] ont étudié l'effet de la région d'entrée sur le transfert de chaleur. Upadhye et Kandlikar [5] ont étudié le refroidissement direct d'une puce électronique par un seul flux de phase d'eau. Nonino et al. [6] ont exploré le transfert de chaleur circulé dans un micro-canal de formes hexagonales, trapézoïdales et rectangulaires; ils ont remarqué que la viscosité dépend étroitement de la température.

\section{Description du modèle de refroidissement proposé}

La Figure 1 représente une vue schématique du modèle proposé (voir tableau 1) suivant le montage illustré sur la figure2.

-La surface totale à refroidir (A.L). -Les dimensions du passage d'écoulement (c.H). - L'ailette (la paroi) séparant les deux canaux est d'une épaisseur ( $\mathrm{t}$ ). - L'épaisseur de la base est (tb); - Les propriétés du fluide de refroidissement et de la matière du dissipateur sont affichées dans le tableau 2.

La température ambiante est égale à $300 \mathrm{~K}$.

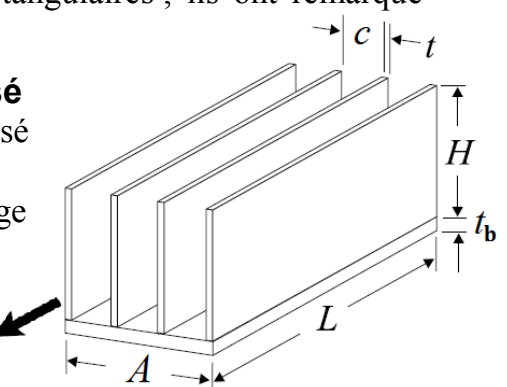

Fig. 1. Géométrie du dissipateur

\begin{tabular}{|c|c|c|c|c|}
\hline $\mathrm{L}$ & $\mathrm{A}$ & $\mathrm{H}$ & $\mathrm{t}$ & $\mathrm{tb}$ \\
\hline 45 & 55 & $01-06$ & $0.1-02$ & $0.5-06$ \\
\hline
\end{tabular}

Tableau 1 : Dimensions du dissipateur (mm)

* Corresponding author: arisaek@yahoo.fr 


\begin{tabular}{|c|c|c|c|c|c|}
\hline & Matière & $\mathrm{g}\left(\mathrm{Kg} / \mathrm{m}^{3}\right)$ & $\mathrm{C}_{\mathrm{P}}(\mathrm{J} / \mathrm{Kg} \mathrm{K})$ & $\lambda(\mathrm{W} / \mathrm{m} \mathrm{K})$ & $\mu(\mathrm{Pa} . \mathrm{s})$ \\
\hline Fluide de base & eau & 997 & 4187 & 0.613 & 0.000855 \\
\hline Matière (dissipateur) & Cuivre & 2330 & 703 & 148 & - \\
\hline
\end{tabular}

Tableau 2 : Propriétés thermiques de l'eau et du Cuivre à une température égale à $300 \mathrm{~K}$

\section{Validation des résultats numériques}

Les effets de plusieurs paramètres sur la température de base " $\mathrm{T}_{\text {base }}$ du dissipateur de chaleur, telles que les dimensions du canal $(\mathrm{c})$ et $(\mathrm{H})$, l'épaisseur de la paroi du canal ( $\mathrm{t}$ ), l'épaisseur de la base inférieure du dissipateur $\left(\mathrm{t}_{\mathrm{b}}\right)$ ainsi que le débit volumétrique du fluide de refroidissement $\mathrm{Q}$. Les calculs sont effectués dans le cas d'une ailette de hauteur $\mathrm{H}=3 \mathrm{~mm}$ d'épaisseur $\mathrm{t}=1 \mathrm{~mm}$, de base inférieure $t_{\mathrm{b}}=3 \mathrm{~mm}$ et pour trois valeurs de débit d'écoulement: $\mathrm{Q}=0,5 ; 0,75$ et $11 / \mathrm{mn}$.

La Figure 3 présente la variation de la température de base $\mathrm{du}$ dissipateur en fonction du débit pour différentes valeurs de l'espacement "c".

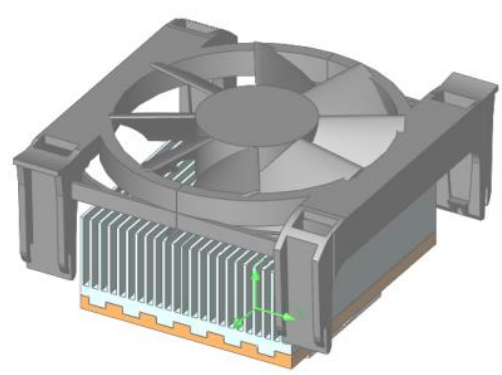

Fig.2 Montage dissipateur+ ventilateur Un bon accord est observé entre les résultats trouvés et les données expérimentales de Jajja et al. Il est important de souligner pour ce cas d'étude; la température la plus basse $: \mathrm{T}_{\text {base }}=41,2{ }^{\circ} \mathrm{C}$ est obtenue pour $\mathrm{Q}=1 \mathrm{l} / \mathrm{mn}$ et $\mathrm{c}=0,2 \mathrm{~mm}$.
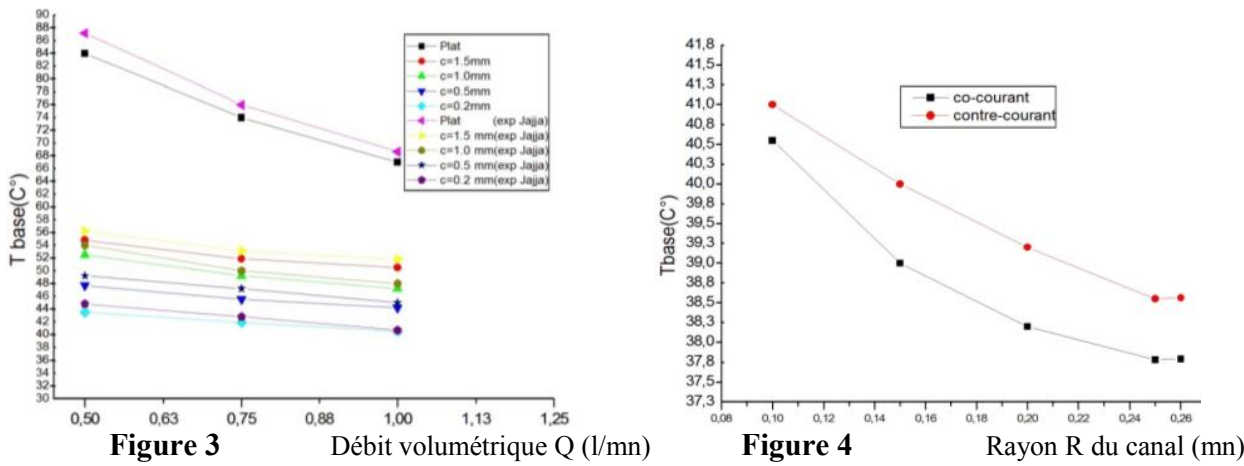

Variation de la température de base en fonction du débit volumétrique et du rayon du canal

La Figure 4 montre la variation de $\mathrm{T}_{\text {base }}$ avec le rayon "R" des canaux circulaires pour un écoulement de fluide en co-courant et contre-courant. Pour le contre-courant et pour les deux valeurs de $\mathrm{R}=0,25 \mathrm{~mm}$ et $0,26 \mathrm{~mm}, \mathrm{~T}_{\text {base }}=37,7^{\circ} \mathrm{C}$. Ainsi, la valeur de $\mathrm{R}=0,25 \mathrm{~mm}$ est choisie comme étant l'optimale. On remarque que la technique de refroidissement par contre-courant est nettement plus performante que celle du co-courant.

\section{Etude expérimentale}

Le montage expérimental (Fig.5) pour valider le modèle numérique et comparer l'efficacité des différents dissipateurs commerciaux utilisés dans plusieurs microordinateurs. Les résultats sont présentés sous forme de contours de températures ainsi que la température mesurée expérimentalement dans certains points du dissipateur.

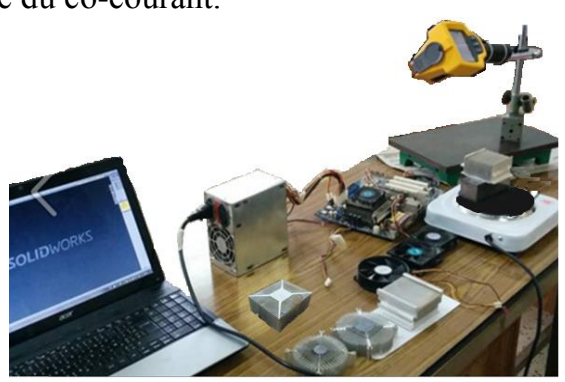

Fig.5 Montage expérimental 


\subsection{Dissipateur avec simple géométrie D1}

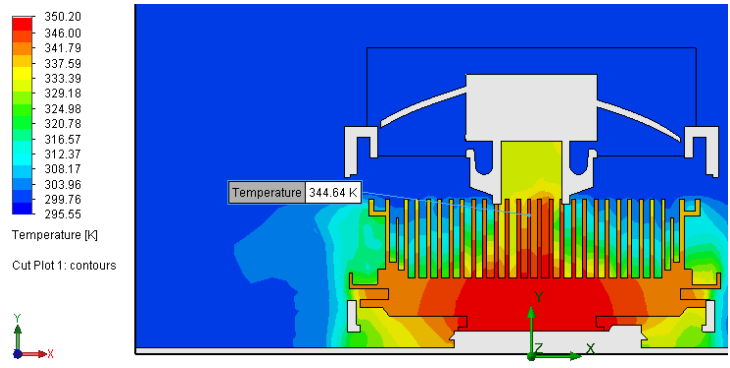

Fig. 6 (a) Champ de températures

\subsection{Dissipateur avec géométrie ronde $D 2$}
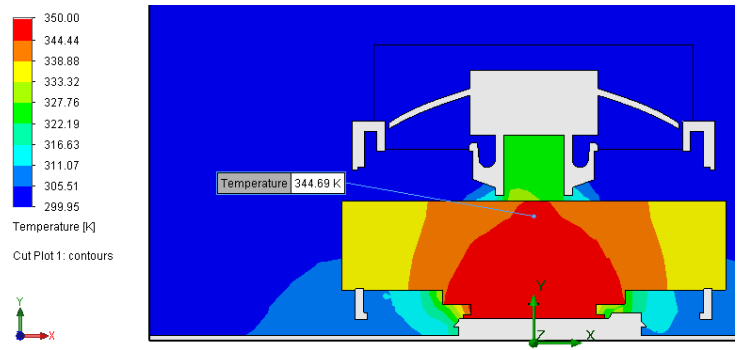

Fig. 7 (a) Champ de températures

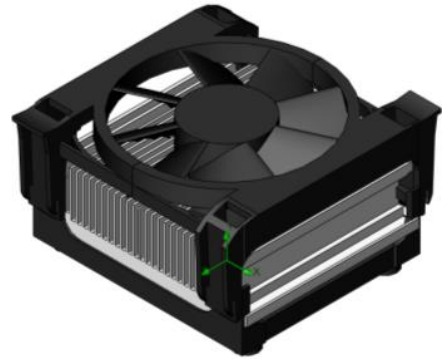

(b) Montage du dissipateur

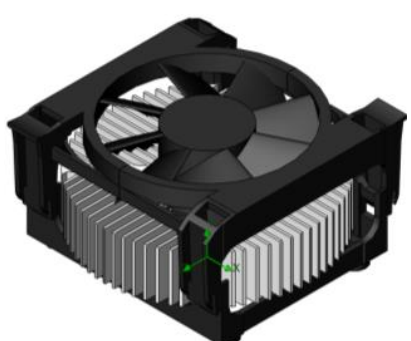

(b) Montage du dissipateur D2+ ventilateur

\section{Conclusion}

Un grand nombre de géométries de dissipateur de chaleur est testé pour plusieurs variantes d'ailette : épaisseur, hauteur, la dimension de la base et le débit volu99métrique.

Pour refroidir un microprocesseur sous une puissance de $325 \mathrm{~W}$, la température la plus basse mesurée est égale à $37,35^{\circ} \mathrm{C}$ est obtenue pour un espacement entre ailettes "c" de 0,2 $\mathrm{mm}$ avec une épaisseur " $\mathrm{t}$ " de $2 \mathrm{~mm}$ et une hauteur " $\mathrm{H}$ " égale à $3 \mathrm{~mm}$ véhiculant un débit de $21 / \mathrm{mn}$.

Dans l'étude expérimentale, les différents dissipateurs sont testé dans les mêmes conditions opératoires (Température ambiante $=300 \mathrm{~K}$ et celle de la source chaude $=350 \mathrm{~K}$ ) afin de déterminer le nombre d'ailettes actives. En prenant compte du critère d'un meilleur transfert de chaleur, le dissipateur optimal sélectionné "D2" est constitué de 44 ailettes de petite forme géométrique ronde.

\section{Références bibliographiques}

[1] A. Capozzoli, G. Primiceri, Cooling systems in data centers: state of art and emerging technologies, Energy Procedia 83 (2015) 484-493.

[2] H. Safikhani, H. Dolatabadi, Multi-objective optimization of cooling of a stack minichannels and conventional channels subjected to natural convection, Appl. Therm. Eng. 96 (2016) 144-150.

[3] M. Steinke, S. Kandlikar, Single-Phase Liquid Friction Factors in Micro-channels, Int. J. Term. Si. 45 (2006) 1073-1083.

[4] P. S. Lee, S. V. Garimella, Thermally Developing Flow and Heat Transfer in Rectangular Microchannels of Different Aspect Ratios, Int. J. Heat Mass Trans. 49 (2006) 3060-3067.

[5] H.R. Upadhye, S.G. Kandlikar, Optimization of micro-channel geometry for direct chip cooling using single phase heat transfer, Proceed. Micro-channels Minichannels, 2004-2398 (2004) 679-685.

[6] C. Nonino, S. Savino, S. Del Giudice, Temperature-dependent viscosity and viscous dissipation effects in micro-channel flows with uniform wall heat flux, Heat Trans. Eng. 31 (2010) 682-691.

[7] S. Ayub Jajja, W. Ali, A. Maryam, Water cooled minichannel heat sinks for microprocessor cooling: effect of fin spacing, Appl. Therm. Eng. 64 (2014) 76- 82. 